IJHG

26,3

Received 25 November 2020 Revised 19 February 2021 Accepted 15 March 2021

\section{Managers' role in supporting resilience in healthcare: a proposed model of how managers contribute to a healthcare system's overall resilience}

\author{
Eline Ree \\ Department of Quality and Health Technology, Faculty of Health Sciences, \\ SHARE - Centre for Resilience in Healthcare, Universitetet $i$ Stavanger, \\ Stavanger, Norway \\ Louise A. Ellis \\ Australian Institute of Health Innovation, \\ Centre for Healthcare Resilience and Implementation Science, \\ Macquarie University, Sydney, Australia, and \\ Siri Wiig \\ SHARE - Centre for Resilience in Healthcare, University of Stavanger, \\ Stavanger, Norway
}

\begin{abstract}
Purpose - To discuss how managers contribute in promoting resilience in healthcare, and to suggest a model of managers' role in supporting resilience and elaborate on how future research and implementation studies can use this to further operationalize the concept and promote healthcare resilience.

Design/methodology/approach - The authors first provide an overview of and discuss the main approaches to healthcare resilience and research on management and resilience. Second, the authors provide examples on how managers work to promote healthcare resilience during a one-year Norwegian longitudinal intervention study following managers in nursing homes and homecare services in their daily quality and safety work. They use this material to propose a model of management and resilience.

Findings - The authors consider managerial strategies to support healthcare resilience as the strategies managers use to engage people in collaborative and coordinated processes that adapt, enhance or reorganize system functioning, promoting possibilities of learning, growth, development and recovery of the healthcare
\end{abstract}

(C) Eline Ree, Louise A. Ellis and Siri Wiig. Published by Emerald Publishing Limited. This article is published under the Creative Commons Attribution (CC BY 4.0) licence. Anyone may reproduce, distribute, translate and create derivative works of this article (for both commercial and non-commercial purposes), subject to full attribution to the original publication and authors. The full terms of this licence may be seen at http://creativecommons.org/licences/by/4.0/legalcode

This article is partly based on our previous research on management and patient safety. The SAFELEAD Primary Care project has received funding from the Research Council of Norway's programme HELSEVEL, under grant agreement 256681/H10, and the University of Stavanger, Norway. The funder has no role in any part of this paper. The authors thank the Research Council Norway for funding the SAFE-LEAD Primary Care project, which made it possible to collaborate on the article. The authors also acknowledge the members of the SAFE-LEAD Primary Care team for their contribution in all phases of the project. Further studies will be continued in the Norwegian research program Resilience in Healthcare (RiH) funded by the Research Council Norway under Grant Agreement Number 275367.

Ethics: The SAFE-LEAD Primary Care project was approved by the Norwegian Centre for Research Data in two phases (phase 1: NSD, ID 52324; phase 2: NSD, ID 54855). Consent to participate is not applicable for this debate article.
International Journal of Health Governance Vol. 26 No. 3, 2021 pp. $266-280$ Emerald Publishing Limited 2059-4631 DOI 10.1108/IJHG-11-2020-0129 
system to maintain high quality care. The authors' model illustrates how managers influence the healthcare systems ability to adapt, enhance and reorganize, with high quality care as the key outcome.

Originality/value - In this study, the authors argue that managerial strategies should be considered and operationalized as part of a healthcare system's overall resilience. They propose a new model of managers' role in supporting resilience to be used in practice, interventions and future research projects.

Keywords Resilience, Managers, Leadership, Healthcare, Quality, Safety

Paper type Conceptual paper
Managers' role in resilience in healthcare

\section{Introduction}

Managers play a significant role in quality and patient safety work and are essential in building a sound patient safety culture. Several management or leadership styles and strategies, including transformational leadership, have been identified as important predictors for quality and patient safety processes and outcomes in health care (Boamah et al., 2018; Wong et al., 2013). However, the literature has identified a dearth of research exploring the role of managers from a healthcare resilience perspective (Grote, 2019; Ledesma, 2014). Managers play an important role in building resilience capacity in employees, and thereby reinforcing organizational resilience (Ledesma, 2014). Most studies on resilience in healthcare have been conducted in inpatient hospital settings, and there is a pressing need for more research in primary care settings, such as nursing homes, homecare and general practice settings (Berg et al., 2018).

An increasing number of elderly people are receiving healthcare services in their homes. Longer life expectancy and increasing numbers of people living with chronic conditions have resulted in higher usage of primary healthcare services (Glette et al., 2018a, b). Within Norway, several white papers, policies and regulations, and action plans exist depicting the need for healthcare services to focus on the role of managers in building good patient safety cultures (Ministry of Health and Care Services, 2016; The Norwegian Directorate of Health, 2019). International research also emphasizes the important role of managers in building a positive patient safety culture (Sammer et al., 2010; Wagner et al., 2018). New developments, reorganizations and changes in the healthcare sector, including technological innovations require the need for healthcare organizations to adapt, and also for managers to play an important role in these adaptions (Guise et al., 2014). Such changes and disruptions can lead to increased risk, errors and disasters, but they can also be an opportunity for development, learning and growth (Wiig et al., 2020b). Recent reviews on resilience in healthcare suggest that in order to advance the field, there is a need for further research on how resilience is distributed at different system levels, including the managerial level (Berg and Aase, 2019; Berg et al., 2018; Ellis et al., 2019; Lim et al., 2020). Specifically, there is a need for more knowledge on how primary care managers work to promote resilience in healthcare, ensuring that the services they lead deliver high quality care during times of disruption and change.

The current paper is a conceptual paper that includes a literature review of selected relevant resilience literature and case presentation of our own published work that results in a proposed model. More specifically, the aims of this conceptual paper are to:

(1) Discuss existing research and approaches to managers' role in supporting resilience.

(2) Provide examples on how managers in Norwegian nursing homes and homecare services contribute to support resilience in healthcare during the implementation of a quality and safety leadership intervention.

(3) Suggest a model for managers' role in supporting resilience that can be used in practice and future research.

To fulfill these aims, we first provide an overview of and discussion of the main approaches to healthcare resilience and research focusing on management and resilience. Second, we provide examples on how managers work to promote healthcare resilience during a one-year 
IJHG

26,3

268
Norwegian longitudinal intervention study (SAFE-LEAD Primary Care), following managers in nursing homes and homecare services in their daily work. The examples were selected purposively to illustrate strategies that potentially promote resilience in healthcare, and therefore do not include an analysis of all aspects of how managers work. The examples are categorized according to the four resilience potentials suggested by Hollnagel (2018) and the characteristics suggested by Berg and Aase (2019). More specifically, we discuss how managers: adapt and adjust to align demands and capacities in their organization in a successful way; monitor or explore the system's function and performance; anticipate upcoming changes or disturbances in their organization and make sense of situations. Finally, we present our model of managers' role in supporting resilience and elaborate on how future research and implementation studies can use this model to promote resilience in healthcare.

\section{The SAFE-LEAD intervention}

The aim of the SAFE-LEAD intervention was to develop, implement and evaluate a researchbased guide (the SAFE-LEAD guide) for managers to guide them in their quality improvement work (Johannessen et al., 2019; Ree et al., 2020a; Wiig et al., 2018). The SAFELEAD guide is centered around the following seven quality challenges that managers often meet in their quality improvement work: structure, culture, competence, engagement, care coordination/organizational politics, physical design/technology and external demands (Johannessen et al., 2019). The guide process consists of three steps, where the management teams start by rating their organization/unit on the quality challenges and agree upon which challenge(s) they want to work on (Step 1). Then they choose specific goals related to meet the quality challenge(s) (Step 2). In the final step, the managers make specific action plans for their quality improvement work related to the selected goals (Step 3).

Four nursing homes and four homecare services in Norway participated in the intervention for approximately six months (Stage 1), while two nursing homes and two homecare services participated over a one-year period (Stage 2). The intervention consisted of workshops with management teams facilitated by the researchers, where the managers applied the SAFELEAD guide in reflections, discussions, planning and implementation of quality improvement initiatives. In addition to workshops, the units in Stage 2 of the intervention received feedback on their quality work from researchers through site-visits on quality meetings and other relevant quality work activities. During the implementation, data was collected by means of interviews, observations, surveys, workshop notes and field notes from site-visits. Based on these data, several papers have been published that are of relevance for the current paper. The previously published papers explore the influence of contextual factors in quality and safety work (Ree et al., 2019), challenges in quality and safety work (Johannessen et al., 2020), reflexive spaces as key for resilience in healthcare (Wiig et al., 2020a), the role of transformational leadership for patient safety culture (Ree and Wiig, 2019b; Seljemo et al., 2020), and how managers use and experience the SAFE-LEAD guide in their quality work (Ree et al., 2020a). Furthermore, it was explored how quality is conceptualized in nursing homes and homecare (Aase et al., 2020), as well as the importance of and challenges related to user involvement (Aase et al., 2020; Ree et al., 2020b). In this conceptual paper, we reflect on the results from these papers from a resilient healthcare perspective, with the aim of exemplifying strategies and activities managers do that potentially support resilience in healthcare.

\section{Main text}

Resilience in healthcare

Resilience is a multidimensional concept, defined and operationalized in many ways depending on research fields and settings (e.g., engineering, psychology, ecology and 
healthcare) (Linnenluecke, 2017; Wiig et al., 2020b; Wiig and Fahlbruch, 2019). Definitions of organizational resilience emphasize aspects such as "coping with unanticipated dangers," "ability to bounce back" (Wildavsky, 1991), and "adapt and recover from disturbances" (Comfort et al., 2010). In healthcare however, the focus is broadened to how resilience manifest itself in the "everyday clinical work" (Hollnagel et al., 2015). Hollnagel et al. (2013) define resilient healthcare as "a health care system's ability to adjust its functioning prior to, during, or following changes and disturbances, so that it can sustain required performance under both expected and unexpected conditions." A recent debate paper from a large international research program on Resilience in Healthcare $(\mathrm{RiH})$ proposed a new working definition of healthcare resilience as "the capacity to adapt to challenges and changes at different system levels, to maintain high quality care" (Wiig et al., 2020b). We apply this definition in this paper. The definition and conceptualization of resilience in the current paper is closely linked to the Resilience Engineering tradition but differs in focusing on the healthcare context specifically with quality as a key outcome of resilient performance. This difference implies seeing safety as one out of several quality dimensions, while resilience engineering is mainly focused on the safety dimension (Wiig et al., 2020a, b). Furthermore, it differs from other definitions in the field in its specific focus on adaptive capacity to change as a basis for high quality care; the ultimate goal of resilient healthcare. The authors argue that quality care consists of four key dimensions: clinical effectiveness, patient safety, care coordination and user involvement. Furthermore, Wiig et al. (2020b) elaborated on the concept of resilience in healthcare as a multilevel phenomenon, emphasizing the importance of collaborative learning and user involvement when adapting to, improving or reorganizing system functioning in response to changes, challenges or disruptions (Wiig et al., 2020b). They suggested the following four key aspects to guide research on resilience in healthcare: (1) What is the purpose of resilience? (for what), (2) what triggers resilience? (to what), (3) what resources are involved? (of what), and (4) what activities and interaction are required for resilient performance? (through what) (Wiig et al., 2020b).

Hollnagel (2018) suggested four important characteristics of resilient organizations: anticipation (knowing what to expect and prepare for), monitoring (knowing what to look for and monitor what happens in and around the system), responding (knowing what to do and adjust to disturbances and changes), and learning (knowing what has happened and learn from experiences). These potentials are interactive and co-dependent, meaning that if an organization fails in one of them, this will also affect the others. A recent literature review of resilient healthcare found four cognitive and behavioral strategies characterizing resilience in organizations: anticipation, sensemaking, adaptation and trade-offs (Berg and Aase, 2019), and these strategies were evident both within and across levels. Similar to the potentials suggested by Hollnagel (2018), these strategies are interrelated. Anticipation is the ability to anticipate and prepare for possible situational changes or hazards before they occur. Adaptations refer to coping with complexity by making adjustments in demanding situations, being flexible, and improvise when necessary. To adapt to new situations or demands, it is necessary to make sense of what is happening, which is referred to as sensemaking (Berg and Aase, 2019). This is related to the concept of trade-offs, which are made between competing goals and tensions. Berg and Aase (2019) postulate two types of trade-offs: cognitive trade-offs (individual level) and trade-offs between competing goals (team level). Anderson et al. (2016) use the CARE model (Concepts for Applying Resilience Engineering) to study resilience in healthcare. The model depicts how Work-As-Imagined (WAI) (alignment between demands and capacity) is constantly adapted because demands and capacity are never fully aligned. These continuous adaptions are referred to as Work-AsDone (WAD) and are crucial to achieve successful or acceptable outcomes in an organization. However, the adaptions made might also be unsuccessful (Anderson et al., 2016). There is limited knowledge on how managers work with these adaptations, as most of the resilient

Managers' role in resilience in healthcare

269 
IJHG

26,3 healthcare studies are conducted at the micro level (e.g., work environmental factors and the adaptions made by frontline clinical staff) (Berg and Aase, 2019).

In the current study, we will provide examples on how managers in nursing homes and homecare services strategize and make adaptations to changes and challenges in their daily work that support resilience. We argue that managers are important resilience actors within healthcare organizations, and we need more insight in how they act to promote resilience in healthcare.

\section{Management and resilience}

Managers are key in facilitating sound patient safety cultures (Sammer et al., 2010). A systematic review concluded that leadership was strongly associated with quality of care outcomes (e.g., mortality, injuries, satisfaction and pain), and that these relationships were evident across different healthcare settings (Sfantou et al., 2017). Transformational leadership behaviors are shown to be especially beneficial in relation to patient safety culture (Clarke, 2013; McFadden et al., 2009; Merrill, 2015; Ree and Wiig, 2019b; Seljemo et al., 2020). Furthermore, transformational leadership is associated with fewer adverse events among patients and greater employee job satisfaction (Boamah et al., 2018). Transformational leaders "broaden and elevate the interests of their employees, generate awareness and acceptance of the purposes and mission of the group, and stir their employees to look beyond their own self-interest for the good of the group" (Bass, 1999, p. 21). Most research on leadership and patient safety have been conducted in hospitals and acute care settings. However, recent results derived from the intervention project on leadership and quality improvement in primary care (SAFE-LEAD), show that transformational leadership is strongly related to better patient safety culture, higher scores on overall perceptions of patient safety, and higher work engagement among employees in Norwegian nursing homes and homecare services (Ree and Wiig, 2019b; Seljemo et al., 2020). Furthermore, qualitative data from managers and employees in the SAFE-LEAD project provides examples on how managers continuously maneuver the surrounding context to adapt to external changes (Johannessen et al., 2020; Ree et al., 2019). Despite the large amount of research on the role of leadership for patient safety practices, cultures and outcomes, there are far less knowledge on how managers contribute in creating resilience in healthcare (Ledesma, 2014). According to Valero et al. (2015), transformational leadership has a significant effect on employees' perceived resilience in organizations. Nevertheless, recent literature reviews on methodological strategies in resilient healthcare studies found only a few or no studies using surveys or mixed methods designs (Berg et al., 2018; Lim et al., 2020). Thus, there is a need for more studies using quantitative methods and mixed methods designs to explore resilience in healthcare.

Managers play an important role in building resilience capacity in employees, and thereby reinforcing resilience in organizations (Ledesma, 2014). As shown by the literature reviewed in the previous section on resilience in healthcare, adaptation is a key aspect. A recent literature review demonstrated that managers plays an important role in creating spaces for ideas to enable adaptive processes (Uhl-Bien and Arena, 2018). Managers have the opportunity to build adaptive capacity in the organization they lead, by helping individuals and teams align the demands they continuously face with capacities, and facilitate sound patient safety cultures (Grote, 2019). Ledesma (2014) argues the need to study the relationship between leadership and resilience. More specifically, how managers affect the organization they lead, the coping skills they use to lead effectively, and how resilience can be fostered within organizations through leadership development (Ledesma, 2014). There is a need for more examples showing how managers work to promote healthcare resilience and use this information for designing possible interventions which is lacking in this field (Ellis et al., 2019; 
Lim et al., 2020; Righi et al.,2015). Based on the definition and conceptualization of resilience in healthcare as used in the current paper, it is meaningful to explore the phenomenon through how managers strategize to adapt to changes and challenging situations in the daily working life. Andersson et al. (2019) argue for the need to study how organizational processes foster traits for organizational resilience in daily organizing, focusing especially on anticipation of disruptive unexpected events to avoid them from happening. Challenges, changes or disruptions to the delivery of care do not have to be a result of serious unexpected events or stressors, but might include situations in the daily working life such as the introduction of new technologies or innovative work practices, challenges in terms of funding, or absence of employees due to sick leave or turnover. In the following sections, we have used such concrete examples from the daily working lives of employees and managers in Norwegian nursing homes and homecare services.

\section{Examples from practice on leadership strategies promoting resilience in healthcare}

Some of the key examples on managerial strategies to support resilience during the SAFELEAD project are summarized in Table 1 and elaborated in the sections below.

Adjustments, adaptations and trade-offs. Managers constantly prioritized and made tradeoffs between competing goals due to misalignments between demands and available resources in their organizations (Johannessen et al., 2020; Ree et al., 2019). Lack of resources, such as time, money and staffing, was a common challenge in both nursing homes and homecare, especially during times of reorganization and change (Johannessen et al., 2020; Ree et al.,2019). To be able to maintain high quality care and keep up with daily quality and safety work, some managers put a lot of effort in to allocating the resources at hand in a strategic and careful way (Ree et al., 2019). They negotiated and collaborated closely within the management team to work creatively within the economic scope they had, to find good solutions and adaptations in challenging situations, and they also involved employees in discussions. For example, they regularly engaged employees in ethical reflections to finding good solutions about scarce resources (Aase et al., 2020). Person-centered care and the involvement of patients and next-of-kin was considered an important part of healthcare quality, and the managers encouraged staff to customize tasks to patients' preferences,

\footnotetext{
Managerial strategies - examples

Allocate resources in a strategic and careful way

Collaborate closely within the management team to find good solutions and adaptations in challenging situations

Engage employees in ethical reflections to find good solutions regarding scarce resources

Use of white-board risk monitoring meetings, boards, reporting systems, competence assessment, and personnel meetings

Staff training and education in all new procedures to ensure confidence in performance

Discuss deviations regularly in management and staff meetings for learning purposes

Use of available networks and resource persons to help the managers make sense of and support implementation of new interventions or changes

Site-visits to see how other organizations implement innovations Delegate responsibility of different tasks and professional issues to employees in order to create accountability, engagement and sense making
}

Resilience characteristics

Adjustments, adaptations and trade-offs

Monitoring and improving system performance

Anticipation and sensemaking

Table 1.

Examples of managerial strategies to support resilience during the SAFE-

LEAD project
Managers' role in resilience in healthcare 
IJHG

26,3 ensuring a bottom-up approach in care (Aase et al., 2020; Ree et al., 2020b). The managers stated that a challenge was to do things differently, to create room for maneuvering.

Anticipation and sensemaking. In order to anticipate forthcoming events and adapt to a new situation or demands, managers used several strategies to help them make sense of what was happening. The managers regularly planned for upcoming changes and used available networks and resource persons in the organization to help them make sense of and support implementation of new interventions or changes (Johannessen et al., 2020; Ree et al., 2019). For example, when implementing a new national dementia plan, they first conducted site-visits to see how other units had solved it. Then they held workshops with the employees to get suggestions and input on what was important for them in the process (Johannessen et al., 2020). Anticipation and sensemaking were necessary before they could adapt to the new situation, which in this case they solved by reallocating resources to create a new position for a dementia coordinator (Johannessen et al., 2020). It was challenging to receive funding for new projects and developments, and since the managers anticipated resistance when requesting more funding in meetings with the municipal manager, they used national guidelines strategically to justify their units' resource needs (Johannessen et al., 2020; Ree et al., 2019).

Furthermore, in planning and adapting to upcoming changes, managers involved employees to ensure a sense of ownership to projects, changes, and interventions. The managers used the strategy of delegating responsibility of different tasks and professional issues to employees in order to create accountability, engagement and sensemaking, and to ensure that new knowledge reached out to all employees (Johannessen et al., 2020; Ree et al., 2019). For example, some employees received additional training, courses, and education in specific professional areas such as nutrition, medical reviews, and diabetes. This saved time for the managers, as well as increased knowledge sharing and sensemaking among employees, and made the organization more prepared for sudden or planned changes. Most of the managers also focused on the "primary contact role," meaning that one employee bore the main responsibility for a patient (Johannessen et al., 2020; Ree et al., 2020b). It was easier to make sense of things happening related to a specific patient when one employee had the full overview of the patient.

The managers always tried to communicate using a language the employees understood and could relate to, in order to make tasks and situations understandable and comprehensible for them. Furthermore, they focused on involving employees in sensemaking. Many engaged a professional development nurse to help them being up to date and to be a driving force in quality improvement work (Ree et al., 2019, 2020a). They highlighted the importance of having professional staff with high competence (Johannessen et al., 2020; Ree et al., 2019). The managers acknowledged that the employees in many cases had more knowledge than they had about things happening in the daily clinical work, and were therefore invaluable in contributing to make sense of situations (Ree et al., 2019). For example, one of the units encountered a lot of resistance when implementing the use of tablets instead of paper work lists. The managers then relied on an employee with good knowledge of the tablets to help other employees make sense of them.

Monitoring and improving system performance. The managers had several strategies and routines for monitoring and exploring the system's function and performance, responding to, and learning. To monitor the systems performance, managers used strategies such as whiteboard risk monitoring meetings, boards, reporting systems, competence assessment, and personnel meetings. The managers emphasized the importance of proper staff training and education in all new procedures to ensure confidence in performance (Aase et al., 2020). Cooperation both within and across the units, and with external actors to exchange experiences was considered important for learning (Aase et al., 2020; Ree et al., 2019). Collecting user experience through surveys and involvement in service development projects in the units and 
organizations was also considered helpful in monitoring system performance. This raised awareness on what they were doing well, what should be improved, and what could be done to improve quality (Ree et al., 2020b).

The managers focused on responding to and learning from errors to avoid repeating them, and the learning potential was considered best immediately after an incident was reported (Johannessen et al., 2020). They regularly discussed deviations in the management meetings for learning purposes, and then addressed them in staff meetings. They were eager to discuss errors and deviations with the employees to find solutions together on what they could do to prevent similar mistakes in the future. Near misses were just as important to discuss as serious events to improve healthcare quality (Aase et al., 2020; Johannessen et al., 2020). They encouraged employees to report, and actively worked against a blame-culture by specifying that everyone makes mistakes and that the aim of addressing them is to learn from them.

Transformational leadership behavior. Previous studies from nursing homes and homecare services show that transformational leadership is a strong predictor of patient safety culture (Ree and Wiig, 2019b; Seljemo et al., 2020), work engagement (Ree and Wiig, $2019 \mathrm{~b}$ ) and person-centered care (Ree, 2020). Transformational leadership was measured in surveys, asking employees to rate their immediate manager on questions representing a global measure of perceived transformational leadership (Carless et al., 2000). The questions captured the following seven transformational leadership behaviors: (1) communicates a clear and positive vision, (2) develops staff, (3) supports staff, (4) empowers staff, (5) is innovative, (6) leads by example, and (7) is charismatic. The studies also show that patient safety culture was a strong predictor for employees' overall perceptions of patient safety (Ree and Wiig, 2019a) and for person-centered care (Ree, 2020) in nursing homes and homecare services.

These findings indicate the mutual relationship between leadership, patient safety culture, and person-centered care. In line with the qualitative findings (Johannessen et al., 2020), they support the role of managers' strategies in shaping, influencing, and acting upon the surrounding context and culture in which quality and safety work are conducted.

How can interventions be used to promote resilience? The role of reflexive spaces. During the SAFE-LEAD intervention period, managers were shadowed on site to observe their work on quality and safety. They emphasized the value of working together on the guide in management teams, as it stimulated joint reflections, and greater awareness about their quality work and challenges (Ree et al., 2020a). Discussing and reflecting in groups made them more pro-active and helped them find good solutions on emerging risks, challenges and changes facing the organizations. This intervention acted as a reflexive space, where managers came together to reflect upon current challenges, adaptations and needs in their daily work (Ree et al., 2020a; Wiig et al., 2020a). It also acted as a bridge between departments as managers met across departments to exchange knowledge, reflections, and action plans, and to agree on new improvement efforts (Ree et al., 2020a; Wiig et al., 2020a). This is an example of how healthcare managers can use reflexive spaces to stimulate resilience in healthcare (Wiig et al., 2020a).

The reflexive spaces can also be broadened to involve actors and stakeholders beyond the management team. For example, at the end of the SAFE-LEAD intervention, the managers in one of the homecare units invited employees to a workshop to work together on how they could further improve quality of care. The way in which the managers proceeded during this workshop and the focus was very much about strengthening resilience in the organization. The main bullet points that the managers listed for discussion in this workshop were these: "what have worked well, and why?" and "what can be better, and how?". The managers welcomed input from all employees, there were good discussions, and at the end of the workshop they made an action plan together with the employees on what they should focus on, what to improve, and how. The workshop incorporated all of the resilience strategies and
Managers' role in resilience in healthcare 
IJHG

26,3

helped us understand possible options and strategies to pursue at the managerial level. This could be of high interest for the resilient healthcare research community as an example of how to think about interventions to promote resilience. The key here is related to establishing conditions under which resilient performance can occur and our examples illustrates the role of manager in this process. This brings us to the modelling aspect elaborated in the next section.

\section{4}

A proposed model of managers' role in supporting resilience

Based on the literature on management and resilience in healthcare discussed in this paper, we propose a model of managers' role in supporting resilience in healthcare (Figure 1). As we have demonstrated, managers are an important part of healthcare systems' capacity to adapt to changes and challenges to maintain high quality care. We further argue that managers are key in supporting resilience in healthcare, and the role of managers should thus be part of the operationalization of the concept. Although many of the examples in our conceptual paper are taken from nursing homes and homecare services, we consider the model to be of relevance to all healthcare services. The model illustrates how managerial strategies are important to adjust, adapt, monitor, anticipate and make sense of new situations, challenges, or disruptions (Figure 1). Managers play an important role for the work environment and safety culture, by prioritizing and allocating available resources with demands, and setting the agenda for the organization's quality and safety work. These processes are central for the system's ability to adapt, enhance and reorganize, and thereby facilitating or hindering healthcare resilience. When the adaptations are successful, the consequence for the organization are learning, growth, development and/or recovery, contributing to better healthcare quality as well as strengthening the organizations adaptive capacity when meeting new situations, challenges and changes in the future (Figure 1). Thus, our model shows how managers contribute to support healthcare resilience through managerial strategies and their impact on the work environment, safety culture and system functioning.

Our proposed model is in line with the recent definition of and conceptualization of resilience in healthcare by Wiig et al. (2020b), which emphasizes the role of patients, users, next-of-kin and other stakeholders as co-creators of resilience and consider healthcare quality as the key outcome for resilience in healthcare (Wiig et al., 2020b). We have demonstrated that involving these actors is an important part of the strategies healthcare managers use to enable the organization and its members to anticipate forthcoming changes, make sense of situations, adapt to changes, and monitor system performance and learn. Managers also interact with networks and resource persons within, across and outside of their unit/ department. They discuss deviations regularly in management and staff meetings, not only serious events but also near misses in order to learn. The managers use reflexive spaces such as management meetings and workshops to reflect upon current challenges, adaptations, and needs, and they facilitate competence development through continuous education and training among the work force. Involvement of staff, competence development and clear communication represent the transformational leadership behaviors of communicating a clear vision, and in developing, supporting and empowering staff (Carless et al., 2000). The strategies managers used are interrelated and are therefore difficult to separate into the resilient potentials suggested by Hollnagel (2018) or into the characteristics suggested by Berg and Aase (2019). The co-dependence of the potentials, meaning that failing in one of them will affect the others, also supports the notion that managerial strategies promoting healthcare resilience affects all of the potentials in different ways. For example, involvement of patients and users is necessary to make sense of situations and anticipate forthcoming changes. At the same time, patient and user involvement informs managers about possible consequences of adaptations and adjustments. Involving patients and users in quality 


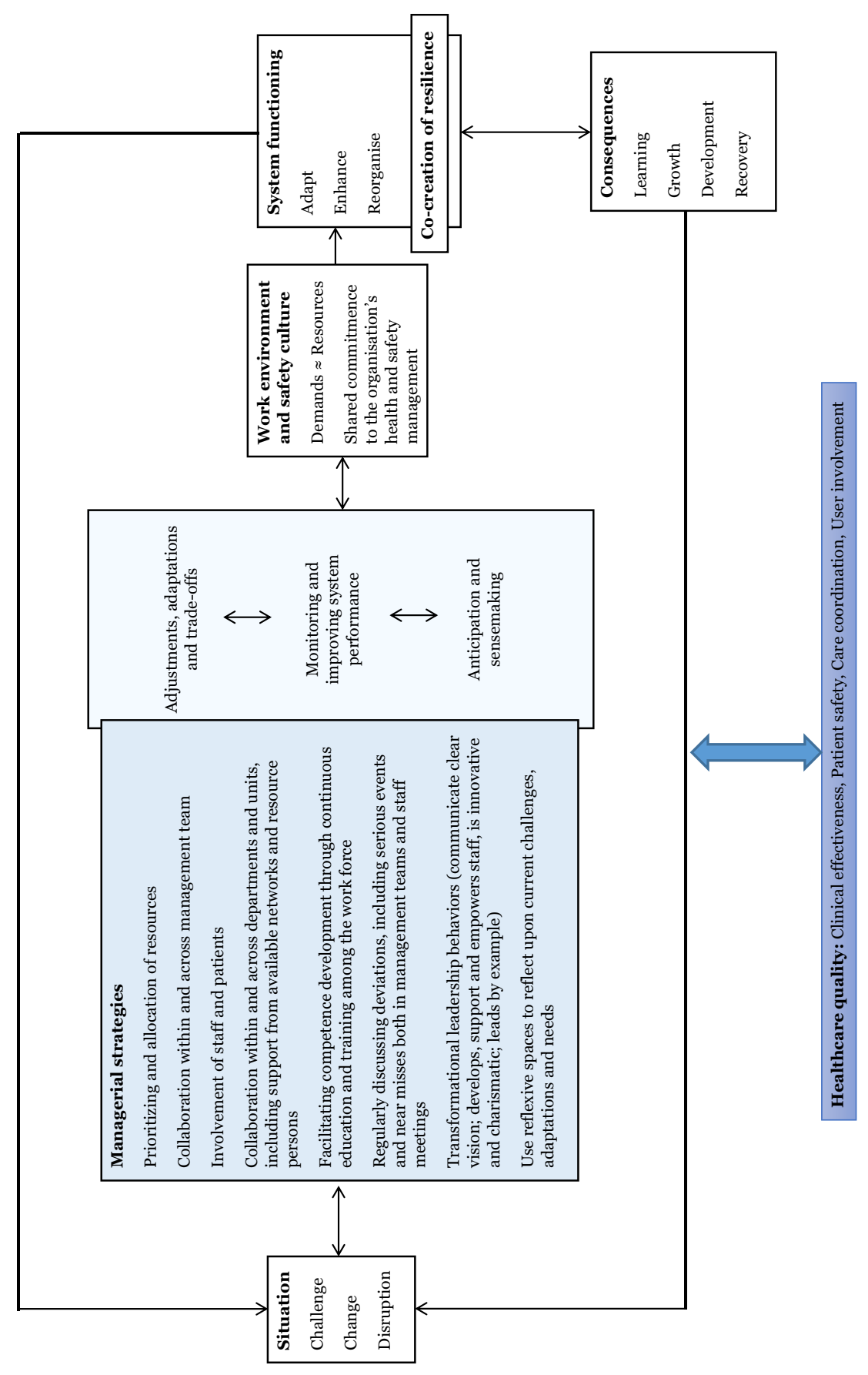

Managers' role in resilience in healthcare

275

Figure 1.

A model of managers role in supporting resilience 
IJHG

26,3 improvement by means of surveys or other measures are important to understand and improve system performance and learn.

Managers have a responsibility and opportunity to act on their surrounding context, make prioritizations and adaptations to ensure a proper balance between demands and available resources and a sound patient safety culture. Thus, the strategies managers use influence their work environment and the patient safety culture. They contribute to a shared commitment among individuals to the organization's overall health and safety management. Therefore, we argue that managers' role in supporting resilience consists of the strategies managers use to engage people in collaborative and coordinated processes that adapt, enhance or reorganize system functioning, promoting possibilities for learning, growth, development and recovery of the healthcare system to maintain high quality care. These capacities of the system allow it to maintain high quality care before, during or after changes, challenges, or disruptions, which is in line with the recent definition of resilience in healthcare (Wiig et al., 2020b). The managerial strategies focusing on involving all individuals in the healthcare system in the processes of acting upon, adjusting, and adapting to their surrounding context, result in co-creation of resilience among all individuals involved in the system, as depicted in our model (Figure 1).

The lack of quantitative studies exploring resilience in healthcare calls for the need to further operationalize the concept, by developing a validated questionnaire based on the theoretical conceptualization and definition of the concept. The proposed model of managers' role in supporting resilience can be used to guide development of a resilience in healthcare questionnaire. Strategies used by managers to promote healthcare resilience should be reflected in measurements of healthcare resilience potentials. Hollnagel (2015) has proposed the Resilience Analysis Grid (RAG) to measure how well a system performs on the four abilities (responding, monitoring, learning, and anticipating). However, we argue that the measurement of healthcare resilience should be adapted to the broader conceptualization and definition proposed by Wiig et al. (2020b). The authors define resilience with high quality care as the main outcome. They include the role of collaborative and coordinated processes in reorganizing system functioning, allowing the system to maintain high quality care during challenges, changes or disruptions. User involvement and clinical effectiveness are also fundamental aspects of their conceptualization of quality, as well as patient safety. Measurement of healthcare resilience should also reveal the adaptive capacity at all levels of the system (i.e., individual, team, management, and system levels). Additionally, a questionnaire of resilience in healthcare should include questions to explore the four core questions in which Wiig et al. (2020b) argue need to be addressed to operationalize and guide resilience in healthcare research: "resilience for what, to what, of what, and through what." Moreover, in line with the proposed model of managers' role in resilience and the resilience conceptualization by Wiig et al. (2020b), the following aspects should be addressed in the questionnaire:

(1) Engagement and involvement of staff, patients, users, next-of-kin, other stakeholders/actors in collaborative and coordinated processes at the individual, team, management, and system level.

(2) Managerial strategies promoting resilience, including transformational leadership behaviors.

(3) Collaborative learning at team and management level.

We suggest that further research is needed to explore ways of operationalizing healthcare resilience in developing measurable questionnaire items based on the definition and conceptualization by Wiig et al. (2020b), and our proposed model of managers' role in supporting resilience. 


\section{Limitations}

The proposed model is only a first step in trying to illustrate the relationship between managerial strategies, resilience, and healthcare quality based on findings from our own studies and relevant previous studies on the topics. Thus, the validity of the model remains to be tested and explored in future studies. A possible limitation is the lack of a systematic literature review of the management and resilience literature. However, there are numerous definitions and conceptualizations of resilience in the research literature, and we wanted to further expand on the definition and conceptualization by Wiig et al. (2020b) in the current paper, and therefore included selected literature relevant for this purpose. The SAFE-LEAD project was not designed as a resilience in healthcare project, but the data material included rich data on how managers constantly strategized and made adaptations in the face of risks, challenges and changes. This contributed to trigger our conceptualization and development of further ideas into modelling mangers' role in resilience.

\section{Conclusion}

In this paper, we have presented our model of managers' role in supporting resilience and suggest that managerial strategies should be considered and operationalized as part of a healthcare system's overall resilience. Our proposed model illustrates how managers influence the healthcare systems ability to adapt, enhance and reorganize, with healthcare quality as the key outcome, consisting of the four dimensions of clinical effectiveness, patient safety, care coordination and user involvement. However, the validity of the model needs to be tested and explored in several healthcare settings, including primary care settings such as nursing homes and homecare services, as well as specialist healthcare such as hospitals and prehospital care settings.

We argue for the need of developing a questionnaire that operationalizes resilience in healthcare and includes measurement of managers' role in supporting resilience. Our model can be used to guide the development of a resilience in healthcare questionnaire. The model can also be used in future resilience studies to systematically explore how managers strategize and adapt to support resilience in healthcare, and thereby contributing to healthcare quality. Managers in different healthcare settings can use the model to guide them in their effort of responding to and adapt to the continuously changing circumstances in their daily working lives. Operationalizing resilience in healthcare and developing a questionnaire based on the suggested aspects is important to gain more knowledge through large quantitative studies that can be used to inform new interventions and implementation studies in this field.

\section{References}

Aase, I., Ree, E., Johannessen, T., Strømme, T., Ullebust, B., Holen-Rabbersvik, E., Thomsen, L.H., Schibevaag, L., van de Bovenkamp, H. and Wiig, S. (2020), "Talking about quality: how 'quality' is conceptualized in nursing homes and homecare challenges in quality and safety work in nursing homes and home care - a case study as basis for theory development”, BMC Health Services Research, Vol. 21 No. 1, pp. 1-12.

Anderson, J., Ross, A., Back, J., Duncan, M., Snell, P., Walsh, K. and Jaye, P. (2016), "Implementing resilience engineering for healthcare quality improvement using the CARE model: a feasibility study protocol”, Pilot and Feasibility Studies, Vol. 2 No. 1, doi: 10.1186/s40814-016-0103-x.

Andersson, T., Cäker, M., Tengblad, S. and Wickelgren, M. (2019), "Building traits for organizational resilience through balancing organizational structures", Scandinavian Journal of Management, Vol. 35 No. 1, pp. 36-45.

Bass, B.M. (1999), "Two decades of Research and Development in transformational leadership", European Journal of Work and Organizational Psychology, Vol. 8, doi: 10.1080/135943299398410. 
IJHG

26,3

Berg, S.H. and Aase, K. (2019), "Resilient characteristics as described in empirical studies on health care", in Wiig, S. and Fahlbruch, B. (Eds), Exploring Resilience - A Scientific Journey from Practice to Theory, Springer Open, Cham, pp. 79-87.

Berg, S.H., Akerjordet, K., Ekstedt, M. and Aase, K. (2018), "Methodological strategies in resilient health care studies: an integrative review", Safety Science, Vol. 110, pp. 300-312.

Boamah, S.A., Laschinger, H.K.S., Wong, C. and Clarke, S. (2018), "Effect of transformational leadership on job satisfaction and patient safety outcomes", Journal of Nursing Outlook, Vol. 66 No. 2, pp. 180-189.

Carless, S.A., Wearing, A.J. and Mann, L. (2000), "A short measure of transformational leadership”, Journal of Business Psychology, Vol. 14 No. 3, pp. 389-405.

Clarke, S. (2013), "Safety leadership: a meta-analytic review of transformational and transactional leadership styles as antecedents of safety behaviours", Journal of Occupational and Organizational Psychology, Vol. 86 No. 1, pp. 22-49, doi: 10.1111/j.2044-8325.2012.02064.x.

Comfort, L.K., Boin, A. and Demchak, C.C. (Eds) (2010), Designing Resilience: Preparing for Extreme Events, University of Pittsburgh Press, Pittsburgh.

Ellis, L.A., Churruca, K., Clay-Williams, R., Pomare, C., Austin, E.E., Long, J.C., Grødahl, A. and Braithwaite, J. (2019), "Patterns of resilience: a scoping review and bibliometric analysis of resilient health care", Safety Science, Vol. 118, pp. 241-257.

Glette, M.K., Kringeland, T., Røise, O. and Wiig, S. (2018a), "Exploring physicians' decision-making in hospital readmission processes-a comparative case study", BMC Health Services Research, Vol. 18 No. 1, pp. 1-12.

Glette, M.K., Røise, O., Kringeland, T., Churruca, K., Braithwaite, J. and Wiig, S. (2018b), "Nursing home leaders' and nurses' experiences of resources, staffing and competence levels and the relation to hospital readmissions-a case study", BMC Health Services Research, Vol. 18 No. 1.

Grote, G. (2019), "Leadership in resilient organization”, in Wiig, S. and Fahlbruch, B. (Eds), Exploring Resilience. A Scientific Journey from Practice to Theory, Springer Open, Cham, pp. 59-67.

Guise, V., Anderson, J. and Wiig, S. (2014), "Patient safety risks associated with telecare: a systematic review and narrative synthesis of the literature", BMC Health Services Research, Vol. 14 No. 1, pp. 1-15.

Hollnagel, E. (2015), RAG-the Resilience Analysis Grid. Resilience Engineering in Practice, A Guidebook Ashgate, Farnham, pp. 275-296.

Hollnagel, E. (2018), Safety-II in Practice: Developing the Resilience Potentials, Taylor and Francis, New York, NY.

Hollnagel, E., Braithwaite, J. and Wears, R. (Eds) (2013), Resilient Health Care, CRC Press, Boca Raton.

Hollnagel, E., Braithwaite, J. and Wears, R.L. (Eds) (2015), Resilient Health Care, Volume 2: The Resilience of Everyday Clinical Work, Ashgate Publishing, New York, NY.

Johannessen, T., Ree, E., Strømme, T., Aase, I., Bal, R. and Wiig, S. (2019), "Designing and pilot testing of a leadership intervention to improve quality and safety in nursing homes and home care (the SAFE-LEAD intervention)", BMJ Open, Vol. 9 No. 6, doi: 10.1136/bmjopen-2018-027790.

Johannessen, T., Ree, E., Aase, I., Bal, R. and Wiig, S. (2020), "Exploring challenges in quality and safety work in nursing homes and home care-a case study as basis for theory development", BMC Health Services Research, Vol. 20 No. 1, pp. 1-12, doi: 10.1186/s12913-020-05149-x.

Ledesma, J. (2014), “Conceptual frameworks and research models on resilience in leadership”, Sage Open, Vol. 4 No. 3, pp. 1-8.

Lim, R., Iflaifel, M., Ryan, K. and Crowley, C. (2020), "Resilient Health Care: a systematic review of conceptualisations, study methods and factors that develop resilience", BMC Health Services Research, Vol. 20, doi: 10.1186/s12913-020-05208-3. 
Linnenluecke, M.K. (2017), "Resilience in business and management research: a review of influential publications and a research agenda", International Journal of Management Reviews, Vol. 19 No. 1, pp. 4-30.

McFadden, K.L., Henagan, S.C. and Gowen, C.R. (2009), "The patient safety chain: transformational leadership's effect on patient safety culture, initiatives, and outcomes", Journal of Operations Management, Vol. 27 No. 5, pp. 390-404, doi: 10.1016/j.jom.2009.01.001.

Merrill, K.C. (2015), "Leadership style and patient safety: implications for nurse managers", Journal of Nursing Administration, Vol. 45 No. 6, pp. 319-324.

Ministry of Health and Care Services (2016), Regulation on Leadership and Quality Improvement in the Health and Care Services [in Norwegian], Ministry of Health and Care Services, Oslo.

Ree, E. (2020), "What is the role of transformational leadership, work environment and patient safety culture for person-centered care? A cross-sectional study in Norwegian nursing homes and homecare services", Nursing Open, Vol. 7 No. 6, pp. 1988-96.

Ree, E. and Wiig, S. (2019a), "Employees' perceptions of patient safety culture in Norwegian nursing homes and home care services", BMC Health Services Research, Vol. 19 No. 1, pp. 1-7, doi: 10. 1186/s12913-019-4456-8.

Ree, E. and Wiig, S. (2019b), "Linking transformational leadership, patient safety culture and work engagement in home care services", Nursing Open, Vol. 7 No. 1, pp. 256-264, doi: 10.1002/ nop2.386.

Ree, E., Johannessen, T. and Wiig, S. (2019), "How do contextual factors influence quality and safety work in the Norwegian home care and nursing home settings? A qualitative study about managers' experiences”, BMJ Open, Vol. 9 No. 7, doi: 10.1136/bmjopen-2018-025197.

Ree, E., Aase, I., Strømme, T., Westbrook, J. and Wiig, S. (2020a), "Lessons learnt from nursing home and homecare managers' experiences with using the SAFE-LEAD guide", Tidsskrift for Omsorgsforskning, Vol. 6 No. 2, pp. 103-118, doi: 10.18261/issn.2387-5984-2020-02-08.

Ree, E., Wiig, S., Braithwaite, J. and Aase, I. (2020b), "To what degree and how do healthcare professionals in nursing homes and homecare practice user involvement? A mixed methods study", Tidsskrift for omsorgsforskning, Vol. 6 No. 2, pp. 119-136, doi: 10.18261/issn.2387-59842020-02-09.

Righi, A.W., Saurin, T.A. and Wachs, P. (2015), "A systematic literature review of resilience engineering: research areas and a research agenda proposal", Reliability Engineering and System Safety, Vol. 141, pp. 142-152, doi: 10.1016/j.ress.2015.03.007.

Sammer, C.E., Lykens, K., Singh, K.P., Mains, D.A. and Lackan, N.A. (2010), "What is patient safety culture? A review of the literature”, Journal of Nursing Scholarship, Vol. 42 No. 2, pp. 156-165, doi: 10.1111/j.1547-5069.2009.01330.

Seljemo, C., Viksveen, P. and Ree, E. (2020), "The role of transformational leadership, job demands and job resources for patient safety culture in Norwegian nursing homes: a cross-sectional study", BMC Health Services Research, Vol. 20, doi: 10.1186/s12913-020-05671-y.

Sfantou, D., Laliotis, A., Patelarou, A., Sifaki-Pistolla, D., Matalliotakis, M. and Patelarou, E. (2017), "Importance of leadership style towards quality of care measures in healthcare settings: a systematic review", Healthcare, Vol. 5 No. 4, doi: 10.3390/healthcare5040073.

The Norwegian Directorate of Health (2019), National Action Plan for Patient Safety and Quality Improvement Work 2019-2023 [in Norwegian], Ministry of Health and Caee Services, Oslo.

Uhl-Bien, M. and Arena, M. (2018), "Leadership for organizational adaptability: a theoretical synthesis and integrative framework", The Leadership Quarterly, Vol. 29 No 1, pp. 89-104, doi: 10.1016/j. leaqua.2017.12.009.

Valero, J.N., Jung, K. and Andrew, S.A. (2015), "Does transformational leadership build resilient public and nonprofit organizations?”, Disaster Prevention and Management, Vol. 24 No. 1, pp. 4-20.

Wagner, A., Hammer, A., Manser, T., Martus, P., Sturm, H. and Rieger, M. (2018), "Do Occupational and patient safety culture in hospitals share predictors in the field of psychosocial working

Managers' role in resilience in healthcare 
IJHG

26,3

conditions? Findings from a cross-sectional study in German university hospitals", International Journal of Environmental Research and Public Health, Vol. 15 No. 10, doi: 10. 3390/ijerph15102131.

Wiig, S. and Fahlbruch, B. (Eds) (2019), Exploring Resilience: A Scientific Journey from Practice to Theory, Springer Open, Cham.

Wiig, S., Ree, E., Johannessen, T., Strømme, T., Storm, M., Aase, I., Ullebust, B., Holen-Rabbersvik, E., Thomsen, L.H. and Pedersen, A.T.S. (2018), "Improving quality and safety in nursing homes and home care: the study protocol of a mixed-methods research design to implement a leadership intervention”, BMJ Open, Vol. 8 No. 3, doi: 10.1136/bmjopen-2017-020933.

Wiig, S., Aase, K. and Bal, R. (2020a), "Reflexive spaces: leveraging resilience into healthcare regulation and management", Journal of Patient Safety. doi: 10.1097/PTS.0000000000000658.

Wiig, S., Aase, K., Billett, S., Canfield, C., Røise, O., Njå, O., Guise, V., Haraldseid-Driftland, C., Ree, E., Anderson, J.E., Macrae, C., Bourrier, M., Berg, S.H., Bergerød, I.J., Schibevaag, L., Øyri, S.F., Sjøseth, S., O'Hara, J., Kattouw, C.E., Kalakou, F.T., Bentsen, S.B., Manser, T., Jeppesen, E. and on behalf of the RiH Team (2020b), "Defining the boundaries and operational concepts of resilience in the resilience in healthcare research program", BMC Health Services Research, Vol. 20 No. 1, doi: 10.1186/s12913-020-05224-3.

Wildavsky, A.B. (1991), Searching for Safety, Transaction Publishers, New Brunskick, Vol. 10.

Wong, C.A., Cummings, G.G. and Ducharme, L. (2013), "The relationship between nursing leadership and patient outcomes: a systematic review update", Journal of Nursing Management, Vol. 21 No. 5, pp. 709-724, doi: 10.1111/jonm.12116.

\section{Corresponding author}

Eline Ree can be contacted at: eline.ree@uis.no

For instructions on how to order reprints of this article, please visit our website:

www.emeraldgrouppublishing.com/licensing/reprints.htm

Or contact us for further details: permissions@emeraldinsight.com 